\title{
NON-REDUCTIVE PHYSICALISM, MENTAL CAUSATION AND THE NATURE OF ACTIONS
}

\author{
MARKUS E. SCHLOSSER \\ University of Bristol
}

\begin{abstract}
Given some reasonable assumptions concerning the nature of mental causation, non-reductive physicalism faces the following dilemma. If mental events cause physical events, they merely overdetermine their effects (given the causal closure of the physical). If mental events cause only other mental events, they do not make the kind of difference we want them to. This dilemma can be avoided if we drop the dichotomy between physical and mental events. Mental events make a real difference if they cause actions. But actions are neither mental nor physical events. They are realized by physical events, but they are not type-identical with them. This gives us non-reductive physicalism without downward causation. The tenability of this view has been questioned. Jaegwon Kim, in particular, has argued that non-reductive physicalism is committed to downward causation. Appealing to the nature of actions, I will argue that this commitment can be avoided.
\end{abstract}

\section{INTRODUCTION}

Non-reductive physicalism about the mental appears to be an attractive position. It is compatible with the widely held assumption that mental properties are multiply realizable (Putnam 1967). It can accommodate the plausible claim that intentional explanations and attributions of mental attitudes have "no echo" in the physical domain (Davidson 1970). And it claims to be compatible with physicalism and a broadly naturalistic worldview. So it is no surprise that non-reductive physicalism has been a standard view in the analytical philosophy of mind, where the commitment to naturalism is widespread and usually taken for granted.

The most important internal objection to non-reductive physicalism is the causal exclusion argument (also known as the supervenience argument). This argument is the topic of a vast amount of literature. It has shaped the mental causation debate and the development of different forms 
of non-reductive physicalism. Jaegwon Kim (1991, 1998 and 2005) has been the most influential and the most persistent proponent of the causal exclusion challenge.

In this paper I will focus on Kim's most recent formulation of the argument, and I will defend non-reductive physicalism by way of a detour to the philosophy of action. First, I will give a brief outline of the basic notions and I will identify an apparent dilemma for non-reductive physicalism that is closely related to the causal exclusion problem. Then I will outline the standard causal account of the nature of actions, which will provide the basis for my response to Kim's challenge. The focus will be on the first stage of Kim's argument, in which it is argued that non-reductive physicalism is committed to downward causation. I will show that this commitment can be avoided for the most important variety of mental causation; namely, the causation of actions by mental events.

\section{Non-REDUCTIVE PHYSICALISM}

It is controversial how, exactly, non-reductive physicalism should be formulated. The following rough characterization, however, is widely accepted and it is sufficiently precise for a fruitful discussion of the view. The two main kinds of reduction are ontological and theoretical reduction. The former concerns concrete entities and properties, the latter is about theories. Non-reductionism about the mental is non-reductionism about mental properties and psychological theories. I will assume that this entails, firstly, that mental properties are not identical with physical properties, and that, secondly, psychological theories are not reducible to physical and other non-mental theories (in the traditional sense of theory reduction). ${ }^{1}$

Physicalism has the following two main components. It holds, firstly, that the physical domain is causally closed. That is to say, roughly, that every physical event that has a sufficient cause has a sufficient physical cause. The second component can be characterized, for our purposes, with a view towards non-reductionism about the mental. It holds that mental properties are ontologically dependent on physical properties: mental prop-

\footnotetext{
${ }^{1}$ The traditional view of theory reduction says, roughly, that a theory $T$ is reducible just in case there is a lower-level theory $T^{*}$ and a set of bridge-laws $L$ such that the laws of $T$ can be derived from the laws of $T^{*}$ in conjunction with the laws in $L$. For a more detailed outline and discussion see, for instance, Kim 1998.
} 
erties exist only if the right physical properties exist, and they are realized and determined by them. This relation of dependence and determination is usually construed in terms of supervenience (we will turn to this further below). Given all this, we can characterize non-reductive physicalism as the conjunction of physicalism and non-reductionism about the mental.

\section{What is Mental CAUSATION?}

All standard versions of non-reductive physicalism are committed to the claim that mental entities are causally efficacious, and it is widely assumed that mental causation is event-causation. Events may be construed as particulars or as instantiations of properties, and it is common to use the terms "events" and "event-causation" as umbrella terms for events and states and for causation by events and states, respectively. Given this, we can say that there is mental causation only if some mental events are causally efficacious in the sense that they stand in event-causal relations with other events. Further, it is often claimed that there is genuine mental causation only if mental events are causally efficacious in virtue of their mental properties (in virtue of instantiating mental properties). And it is usually assumed that genuine mental causation requires that mental events do not merely overdetermine their effects. Given all this, we can begin with the following three necessary conditions on mental causation:

(1) Mental events are causally efficacious: mental events cause events.

(2) Mental events are causally efficacious in virtue of their mental properties.

(3) Mental events do not merely overdetermine their effects.

One important question has been left open. What kinds of things must mental events cause for there to be genuine mental causation? Are the conditions (1)-(3) jointly sufficient for genuine mental causation, or do we need to impose further restrictions on what kinds of events must be caused?

The contemporary mental causation debate, it is sometimes claimed, has its roots in Donald Davidson's seminal paper "Mental events" (1970). One of the basic assumptions in this paper says that there is interaction between mental and physical events: some physical events cause mental events and some mental events cause physical events (p. 208). Damage to 
muscle tissue, for instance, causes pain, and intentions cause behaviour. Most philosophers have followed Davidson on this. They have assumed, in particular, that mental causation requires what we can call mental-to-physical causation: the causation of physical events by mental events. ${ }^{2}$

However, most philosophers would also acknowledge that mental events can be causally efficacious by causing other mental events. So why insist on interaction with physical events if there can be mental causation within the domain of the mental itself? We can identify two closely related reasons for the insistence on mental-to-physical causation. If there is mental-to-mental causation that satisfies our conditions (1)-(3), then the mental is causally efficacious. Nevertheless, we tend to think that this alone falls short of genuine mental causation, because we tend to think that the mental is truly efficacious only if it causes also physical events. We tend to think that mental events make a real difference only if they make a difference in the physical world. This, in turn, is motivated by the very plausible intuition that an agent's mental events make a real difference only if they make a difference to the agent's overt behaviour. We tend to think that there is genuine mental causation only if mental events cause bodily movement.

\section{A Dilemma for Non-Reductive PhysicAlism}

Let us assume, for the sake of argument, that non-reductive physicalism is true: psychological theories are not reducible, mental properties are not identical with physical properties, but they are dependent on, realized and determined by them. Most philosophers, as just pointed out, think that there is genuine mental causation only if some mental events have physical effects. But mental-to-physical causation leads to the following wellknown problem for non-reductive physicalism. If mental events cause physical events, then they merely overdetermine their effects due to the causal closure of the physical. Assume, for instance, that an agent's decision causes the execution of a bodily movement. Given that this is a physical event, and given the causal closure of the physical, the movement has a sufficient physical cause. The decision, it seems, is neither identical with nor a part of this sufficient cause, as it is not identical with any physical

\footnotetext{
${ }^{2}$ To name only a few, see Kim 1991, 1998 and 2005, Crane 1995 and Menzies 2003.
} 
event. So it seems that the decision merely overdetermines (or "overcauses") the movement. ${ }^{3}$

Given all that, non-reductive physicalism appears to face the following dilemma. If mental events cause physical events, they merely overdetermine their effects, and if they cause only other mental events, they are not truly efficacious. So, either way, the efficacy of the mental falls short of genuine mental causation.

This dilemma is based on a dichotomy between mental-to-physical and mental-to-mental causation. It is based, in other words, on the assumption that mental causation is either mental-to-physical or mental-to-mental - there is no further possibility. I will now argue that this is a false dichotomy.

\section{ACTIONS AND MOVEMENTS}

The causation of actions is, arguably, the most important variety of mental causation. Given this, we can say that there is genuine mental causation if mental events cause actions in a way that satisfies the conditions (1)-(3): there is genuine mental causation if mental events cause actions in virtue of their mental properties and without overdetermination.

Actions can be distinguished from bodily movements. This distinction is familiar within the philosophy of action. But in the philosophy of mind it is often overlooked or neglected, and it is frequently blurred by talk about behaviour. So, what are actions? Are they physical or mental events?

Most philosophers of mind presuppose, implicitly perhaps, an eventcausal theory of action. According to this view, actions are constituted by events with a certain causal history. Certain events, that is, constitute actions in virtue of being caused by the right antecedents (and in the right way). ${ }^{4}$ The right antecedents are mental events that rationalize the action (such as desires, beliefs and intentions). So, on this view, an event constitutes an action only if it is caused by rationalizing mental events, and if it

\footnotetext{
${ }^{3}$ This is, basically, the causal exclusion problem. The causal sufficiency of the physical excludes the causal relevance of the mental. Compare, again, Kim 1991, 1998 and 2005, Crane 1995 and Menzies 2003.

${ }^{4}$ The right way of causation is non-deviant causation. It is widely believed that it cannot be specified what non-deviant causation consists in, and many philosophers think that this is a very serious problem for the causal theory. I do not share this pessimistic assessment, and I have proposed an account of non-deviant causation elsewhere (Schlosser, 2007).
} 
does constitute an action, it does so in virtue of being caused by them. The causal history is therefore part of an action's essence or identity. Actions, in other words, are etiological phenomena, just like banknotes, sunburns or Picasso's paintings. ${ }^{5}$ (A perfect copy of a banknote, for instance, is not a banknote because it has not been printed in the right way, by the right institution. Its causal history is part of its identity.)

It is very plausible to think that some actions are mental actions. On the causal theory, mental actions are realized by and perhaps tokenidentical to mental events. But they are not type-identical with mental events, because being of a certain mental event-type does not determine whether or not the event is an action. The formation of an intention, for instance, may be an action. We form some intentions actively, but others are formed passively. Whether or not a mental event is or constitutes an action depends on its causal history. It is an action only if it is caused by desires, beliefs and intentions in the right way.

The same holds for overt actions (actions that involve bodily movement). Consider the basic action of raising one's arm. Actions of this type are physically realized by bodily movements (arm risings), and perhaps every particular action of this type is token-identical with a particular movement. But they are not type-identical. It is not the case that you are raising your arm whenever your arm rises. The occurrence of the particular type of movement does not determine whether or not the event constitutes an action. The movement, rather, is an action only if it is caused by the right antecedents and in the right way.

What is the rationale behind thinking of actions in etiological terms? Consider the following two widely accepted doctrines. According to the first, all actions are intentional under some description. In particular, something is an action, or counts as an action, only if it is something that is done intentionally under some description (compare Anscombe 1957 and Davidson 1963). According to the second, something is done intentionally only if it is done for reasons (in the minimal sense of being motivated by mental states and events that rationalize its performance). Proponents of the causal theory have argued that the best explanation of the fact that an action is done for reasons is provided by the assumption that it is caused by the mental antecedents that rationalize it (Davidson 1963). Given all this, we can see why the causal history enters into the essence of actions. Some-

\footnotetext{
5 This point is not often made explicit, but it is part of the causal theory of action. Compare Mele 1997.
} 
thing $i$ an action, or counts as an action, in virtue of being caused by rationalizing mental states and events.

A further reason for thinking that actions are not type-identical with the physical events that realize them is given by the fact that most of our non-basic actions are multiply realizable. Suppose that I give someone a signal by raising my arm. In this case, I perform the non-basic action of giving a signal by performing the basic action of raising my arm. Clearly, there are many different ways in which I can give someone a signal. The act-type of giving a signal is in this sense multiply realizable, and therefore not identical with a certain type of movement.

To summarize, according to the standard causal theory, actions are neither mental nor physical events. Given this, there can be genuine mental causation that is neither mental-to-physical nor mental-to-mental causation; namely, mental causation of actions. On this view, actions are caused and causally explained by mental events, and both mental events and actions are realized by physical events. Given, however, the irreducibility of the mental, mental events are not type-identical with physical events, and given the etiological nature of action, actions are not type-identical with physical events. In particular, overt actions are not type-identical with bodily movements. Given further that mental events cause actions in virtue of their mental properties, we obtain a view according to which the mental is causally efficacious in a way that avoids the mentioned dichotomy. Mental events cause actions in virtue of their mental properties and without overdetermination. This is non-reductive physicalism without downward causation (without, that is, mental-to-physical causation).

In the following sections, I will turn to Kim's challenge, according to which non-reductive physicalism is committed to downward causation. But before that, let me add a few brief remarks concerning the interaction between mind and body. I suggested that there is genuine mental causation if mental events cause actions, and that actions are neither mental nor physical events. But what are they? Actions are realized by physical events, and it is plausible to assume that particular act-tokens are identical with physical event-tokens. Despite that, they belong to the domain or level of intentional explanation and mental state attribution. Actions are explained in terms of the agent's reasons (or in terms of rationalizing mental states and events), and we recognize or identify something as an action only under its intentional description.

What about the other direction? Is pain, for instance, caused by physical events? This appears to be undeniable. But consider the following al- 
ternative. It seems plausible to suggest that damage to muscle tissue, for instance, causes certain physical events in the brain that realize the mental event of being in pain. On this view, no physical event causes pain. Physical events cause other physical events, which realize pain. If something like this holds for all mental events, and if the proposed view of mental causation is correct, then there is no causal interaction between the mental and the physical. Nevertheless, epiphenomalism is false, because mental events do cause actions.

\section{OVERDETERMINATION AND DOWNWARD CAUSATION}

One apparent advantage of the suggested non-reductive view is that it avoids the causal exclusion problem. Mental events do not cause physical events. Hence there is no problem of overdetermination and causal exclusion. But it has been argued, most prominently by Jaegwon Kim, that nonreductive physicalism is committed to downward causation. This argument constitutes the first stage in Kim's most recent statement of the causal exclusion argument (2005, pp.39-41). In this first stage, Kim considers the case of mental-to-mental causation. Assume that the mental event $M$ causes another mental event $M^{*}$. According to any version of non-reductive physicalism, $M$ and $M^{*}$ are realized and determined by physical events (their supervenience bases). Assume that $P$ is the supervenience base of $M$ and that $P^{*}$ is the supervenience base of $M^{*}$.

What, Kim asks, explains the occurrence of $M^{*}$ ? There are two candidates: the occurrence of $M$ and the occurrence of $P^{*}$. Both explain the occurrence of $M^{*}$, and together they overdetermine $M^{*}$, albeit not causally. $M$ determines $M^{*}$ in virtue of being its cause, and $P^{*}$ determines $M^{*}$ in virtue of being its supervenience base. This, Kim argues, creates a prima facie tension between two competing explanations, and he suggests that the best way to resolve this tension is to assume that $M$ causes $M^{*}$ by causing its supervenience base $P^{*}$. This shows, according to Kim, that "mental-tomental causation entails mental-to-physical causation - or, more generally, that 'same-level' causation entails 'downward' causation" (p.40). This is represented by the following figure, where the arrows stand for causation and the vertical lines for the relation of realization: 


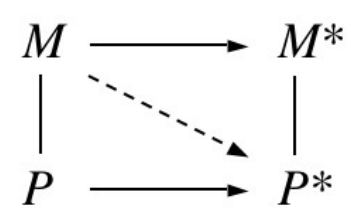

Fig. 1 Mental-to-mental

It would seem obvious that the same reasoning can be applied to the view that I have proposed. If actions are caused by mental events, and if they are realized by physical events, then the resulting tension and overdetermination appears to commit us to downward causation: we must assume that mental events cause the physical events that realize actions.

But this line of reasoning is mistaken. According to Kim, the occurrence of the supervenience base of a certain mental event realizes, determines and necessitates the occurrence of the mental event. So, in the example, the occurrence of $P^{*}$ realizes, determines and necessitates the occurrence of $M^{*} . P^{*}$ by itself necessitates $M^{*}$, as Kim says, "no matter what happened before" (p.39); in particular, no matter whether $M$ occurred or not (unless, that is, $M$ is a cause of $P^{*}$ ). Putting aside the question of whether non-reductive physicalism is in fact committed to this, the same reasoning does not hold for actions.

Actions, we assume, are realized by physical events, such as bodily movements. But the occurrence of a certain bodily movement does neither necessitate nor determine the occurrence of a certain type of action, for the reasons given above. A certain physical event, such as a certain bodily movement, realizes an action only if it has the right causal history. Let us replace the mental event $M^{*}$ by an action of type $A$ :

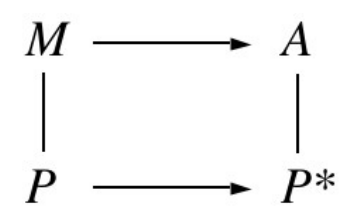

Fig. 2 Mental-to-action

Given the assumptions, $A$ is caused by $M$ and realized by $P^{*}$. But given the causal account of the nature of action, it is not the case that the occurrence of $P^{*}$ necessitates or determines the occurrence of $A$. Whether or not $P^{*}$ realizes $A$ depends on the causal history. 
Given that an action is performed, we can assume that $M$ is a rationalizing cause of $A$. Had there been no rationalizing cause, there would have been no action performed and $P^{*}$ would not have realized an $A$-ing. So, in this particular case, whether an $A$-ing is performed or not depends on whether or not $M$ occurs and on whether or not $M$ causes $A$. Hence, $P^{*}$ alone neither necessitates nor determines the occurrence of $A$. Subsequently, there is no tension due to overdetermination that has to be resolved, and so there is no need to assume that the mental event $M$ must cause $A$ by causing $P^{*}$. The argument, in other words, collapses if the effect of mental causation is an action.

One possible objection to this goes as follows. Given the standard view of the nature of actions, it is not $P^{*}$ alone that necessitates and determines $A$, but $P^{*}$ 's being caused by $P$. So, $P$ 's causing $P^{*}$ is the causal history that makes $P^{*}$ an action. In that way, the occurrence of $A$ is realized and determined by the occurrence of physical events and physical causation, and the problem of overdetermination reappears: $M$ merely overdetermines $A$, as $A$ is determined by $P^{\prime}$ 's causing $P^{*}$.

This objection overlooks the important point that $P^{\text {'s causing }} P^{*}$ realizes and determines the performance of $A$ only if it realizes and determines $M$ 's causing $A$. Non-reductive physicalism is motivated by the thought that rationality and reason-explanation have "no echo" in the physical domain. $P$ does not rationalize $P^{*}$. $P$ cannot rationalize anything, because only mental events can possibly rationalize other events and actions. And mental events, we assume, are not reducible to physical events. Moreover, the fact that $P^{\prime}$ 's causing $P^{*}$ realizes and determines $M$ 's causing $A$ does not raise a problem, as it does not result in the overdetermination of $A$ 's occurrence. If $P$ had not realized $M$ and if $P^{\prime}$ s causing $P^{*}$ had not realized $M$ 's causing $A$, then $P^{\prime}$ 's causing $P^{*}$ would not have realized and determined the occurrence of $A$. In other words, $P$ 's causing $P^{*}$ does not by itself determine $A$. Whether it does depends on whether it also realizes and determines $M$ 's causing $A$. So, $M$ and $P$ 's causing $P^{*}$ do not overdetermine the performance of $A$ - not in any clear and familiar sense of overdetermination. (To be sure, the fact that $P$ 's causing $P^{*}$ realizes and determines $M$ 's causing $A$ is not a problem, because this is precisely what non-reductive physicalism says: the mental is realized and determined by the physical.) 
The objection, however, highlights a more serious issue. Mind-body supervenience is at the core of Kim's characterization of non-reductive physicalism. For the case of mental-to-mental causation Kim assumes, with nonreductive physicalism, that $P$ is the supervenience base of $M$, and that $P^{*}$ is the supervenience base of $M^{*}$. The argument is based on the assumption that non-reductive physicalism shares this commitment to mind-body supervenience. But the response that I have offered appears to violate this presupposition. It claims that the occurrence of $P^{*}$ alone does not determine the occurrence of the effect (on the basis of considerations concerning the nature of actions). This means, firstly, that the response does not share one of the background assumptions that Kim assumed with nonreductive physicalism (and for the sake of argument). And it shows, secondly, that the response is based on the further assumption that the supervenience base of an event may be distinct from the realization base of that event. This assumption appears to be in need of justification. It is not shared by Kim's challenge, nor is it made by standard versions of nonreductive physicalism.

First of all, the distinction between the supervenience base and the realization base of a higher-level event is not entirely implausible or unmotivated. If externalism about mental content is true, for instance, then the supervenience base of some mental events includes the agent's environment and causal history. Despite this, it would still seem plausible to think that the instantiation of a mental event of this kind is realized by the instantiation of some of the agent's intrinsic physical properties. It would seem plausible, in other words, to distinguish the supervenience base from the realization base of the mental event. This distinction, however, raises a host of difficult and controversial issues. I shall therefore propose the following reformulation of my response, which does not presuppose the distinction between the supervenience and the realization bases of mentallevel events.

Let us return to our schema, where a mental-level event $M$ causes a mental-level effect, and where both events have a physical supervenience base $P$ and $P^{*}$, respectively. According to Kim's construal of mental causation, the physical-level and mental-level events occur simultaneously: $P$ occurs exactly when $M$ occurs, and $P^{*}$ occurs exactly when $M^{*}$ (or $A$ ) occurs. In particular, $P^{*}$ determines the effect no matter what happened before. I argued that it does matter what happened before, when the mental- 
level effect is an action. But we do not have to put it that way. We can, instead, assume with $\mathrm{Kim}$ that $P^{*}$ is the supervenience base of the effect, no matter what the effect is, and that the occurrence of $P^{*}$ determines the effect's occurrence, no matter what happened before. We can say this, because we can construe $P^{*}$ as containing the supervenience base of the action's mental antecedent. We can, in other words, think of $P^{*}$ as encompassing both the physical event that realizes and determines the mental antecedent and the bodily movement. ${ }^{6}$ This is represented in the following figure:

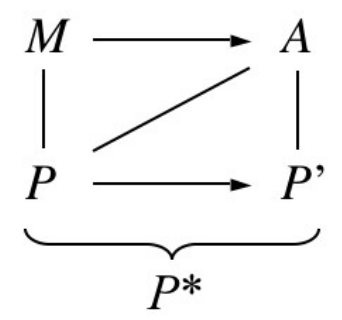

Fig. 2.1 Mental-to-action

Reconstructed in this way, the bodily movement $P^{\prime}$ is part of the action's supervenience base, and so we can say that it partly realizes the action. If we think of it this way, we do not violate the initial assumption that $P$ is the supervenience base of $M$ and that $P^{*}$ is the supervenience base of the mental-level effect that is caused by $M$.

Construed in this way, the response is now in conflict with different presuppositions of Kim's argument. Most obviously, $P$, the supervenience base of the mental antecedent, is not a cause of the effect's supervenience base $P^{*}$ anymore. But this is not problematic as we focus exclusively on the first stage of Kim's argument. The occurrence of $P$ does not play any role in this stage, as its dialectic stems from the purported overdetermination of the effect by $M$ and $P^{*}$ alone. The assumption that $P$ causes $P^{*}$ does not enter Kim's argument before the second stage (compare p.41).

More importantly, though, the reformulation of the response is now in conflict with Kim's definition of mind-body supervenience, which goes as follows:

\footnotetext{
${ }^{6}$ An alternative is to identify the action with the causal process rather than with the effect or product of this process. There are, to my knowledge, no decisive arguments for or against this process view, but I do not want to presuppose this non-standard view of agency here.
} 
Supervenience: Mental properties strongly supervene on physical properties. That is, if any system $s$ instantiates a mental property $M$ at $t$, there necessarily exists a physical property $P$ such that $s$ instantiates $P$ at $t$, and necessarily anything instantiating $P$ at any time instantiates $M$ at that time. (With minor alterations from Kim, 2005, p.33)

According to Kim, mind-body supervenience is a crucial component of non-reductive physicalism. Above, we agreed with this when we assumed that the kind of dependence and determination that holds between physical and mental properties can be captured in terms of supervenience. But whether or not we can agree with Kim's more specific claim depends on how supervenience is defined.

Given the standard causal view on the nature of action, it is clear that we cannot accept Kim's definition of supervenience - at least not for mental-to-action causation. First of all, we need to interpret the definition in a way that covers both mental events and actions. This is not problematic. According to the offered causal account, actions are realized by, dependent on and determined by physical events, just like mental events. Although realized by physical events, actions belong to the mental level, as explained above. In the light of this, Kim's definition of supervenience could be interpreted as covering mental events and actions. It could be interpreted as a claim about the supervenience of mental event-types, mental act-types, and overt act-types (this will be made explicit below).

The problem with Kim's definition is that it presupposes a synchronic occurrence of mental-level events and their corresponding physical supervenience bases. Given the offered account of action, we have good reason to reject this. On that view, a mental or overt action is realized and determined by a physical process that begins before the performance of the action. This just means that the physical supervenience bases of actions begin to occur before the actions begin. (In many cases the time lag may be very short - so short, perhaps, that it cannot be noticed consciously.)

Both mental and physical events take some time to occur. Let us say that a time interval $t^{*}$ includes the interval $t$ just in case $t$ begins at the same time or after $t^{*}$ and ends at the same time or before $t^{*}$. We can then reformulate Kim's definition as follows:

Supervenience*: Mental-level properties (mental event-types, mental act-types, and overt act-types) strongly supervene on physical properties. That is, if any system $s$ instantiates a mental-level property $M$ at $t$, there necessarily exists a physical property $P$ such that $s$ instantiates $P$ 
at $t^{*}$, where $t^{*}$ includes $t$, and necessarily anything instantiating $P$ at a time $t_{P}$ instantiates $M$ at a time $t_{M}$ that is included in $t_{P}$.

Is this move justified? Can we simply define supervenience in a way that suits the response? This move, I think, is justified, and there are no obvious reasons to think that it is begging the question against Kim's objection.

Firstly, there is no obvious reason to think that non-reductive physicalism is committed to Kim's definition of supervenience. The debate on the different notions and definitions of supervenience is complex and intricate. One of the main questions in this debate is how the different definitions of individual supervenience relate to global supervenience. Kim's definition is a version of strong individual supervenience. It concerns, that is, the correlation between the properties of individuals (agents or systems) rather than the global correlations between families of properties within possible worlds. Some philosophers have argued that physicalism can be characterized in terms of global supervenience (Lewis 1983 and Jackson 1998, for instance). Kim argued, at one point, that global supervenience and strong individual supervenience are equivalent (compare Kim 1991). But this is controversial. Arguably, strong supervenience implies global supervenience. But it has been argued that global supervenience does not imply strong individual supervenience (see McLaughlin 1995, pp.37-38, for instance). If this is correct, and if physicalism can be characterized in terms of global supervenience, then non-reductive physicalism is not committed to any version of strong individual supervenience (neither to Kim's Supervenience, nor to Supervenience*). If, on the other hand, the characterization of physicalism requires a form of strong individual supervenience, it still remains to be shown that it requires Kim's Supervenience (rather than the proposed Supervenience*). In connection with that it should be noted that supervenience is not necessarily a synchronic relation, and that the general debate on supervenience does not provide direct support for Kim's time-indexed version of strong individual supervenience, as the versions of strong supervenience discussed within that debate are usually not time-indexed (compare McLaughlin 1995).

Secondly, the question of how the timing of mental events is related to the timing of their neural correlates is largely an empirical question. Benjamin Libet, for instance, argued on the basis of empirical evidence that unconscious brain processes precede both bodily movements and their conscious mental antecedents. In fact, Libet argued that conscious awareness occurs in general a short time after the onset of the corresponding 
neuronal processes (Libet 1996, for instance). Now, even if this is correct, it does not show that all mental events occur after the onset of their neural correlates (because it does not show that unconscious mental events occur after the onset of their neural correlates). Nevertheless, the experimental findings remind us that processes of realization usually take time, and they lend some credibility to the assumption that all mental events may be preceded by their neural correlates. Given all this, Kim's definition of supervenience, which stipulates a strict synchronic relationship between mental events and their supervenience bases, may well turn out to be false.

Thirdly, and most importantly, Kim's definition of supervenience, together with his schema of mental causation, is incompatible with the etiological nature of action. The outlined account of the nature of action is an integral part of the widely accepted causal theory of action, and it is, as I have suggested, plausible on independent grounds. Given this, the proposed revision of the definition of supervenience (Supervenience*) is well motivated and justified.

\section{CONCLUSION}

The argument of this paper leaves us with a somewhat awkward position. According to Kim, non-reductive physicalism is committed to downward causation. I argued that this does not hold for the case of mental-to-action causation. Given my arguments, non-reductive physicalism is not committed to downward causation, if we assume that mental causation is mentalto-action causation. But why would we make this assumption? The causation of intentional actions by mental states and events is perhaps the most important variety of mental causation. But mental events cause other mental events as well as actions, and it seems clear that not all instances of mental-to-mental causation constitute mental agency. The formation of a judgement, for instance, may or may not be a mental action; the occurrence of a memory may cause the formation of a belief without the agent's doing anything; and so on.

So, the argument of this paper provides only a partial defence of nonreductive physicalism. This argument, it should be noted, might hold for essentially etiological properties in general (it might hold, that is, for all higher-level properties with a historical identity or nature). But this seems to be irrelevant, as mental events do not in general fall under this category. It has been suggested that all higher-level properties, and hence all mental- 
level properties, are functional properties (properties that can be individuated in terms of their characteristic causes and effects. Compare, for instance, Shoemaker 1980). But functional properties are not necessarily etiological properties. Consider the mental property of being in pain. If this is a functional property, then it can be characterized in terms of its causes and effects, and it can, presumably, be multiply realized. It can, that is, be realized by any physical event that can play its characteristic causal role. Assume, then, that being in pain is realized in humans by physical events of type $P$ (firings of certain patterns of neurons, for instance). Given this, the instantiation of $P$ in humans is sufficient for the instantiation of the mental property of being in pain. That means that a particular instantiation of pain does not necessarily have to be caused by one of the characteristic causal antecedents of pain. Given this, being in pain is a functional, but not an etiological property.

To conclude, the scope of my argument is restricted to mental-toaction causation. Nevertheless, it highlights some interesting and important features of the problem of mental causation. It shows that an abstract and general treatment of the causal exclusion problem is problematic, and that there are important differences between mental-to-physical, mental-tomental and mental-to-action causation. And it highlights the fact that it is important to distinguish clearly between bodily movements and actions.

\section{REFERENCES}

Anscombe, G. (1957) Intention, Cambridge: Harvard University Press.

Crane, T. (1995) "The mental causation debate", Proceedings of the Aristotelian Society, Supplementary Volume LXIX, pp.211-236.

Davidson, D. (1970) "Mental events", reprinted in his Essays on Actions and Events, Oxford: Clarendon Press (1980), pp.207-227.

Davidson, D. (1963) “Actions, reasons, and causes", reprinted in his Essays on Actions and Events, Oxford: Clarendon Press (1980), pp.3-20.

Jackson, F. (1998) From Metaphysics to Ethics, Oxford: Oxford University Press.

Putnam, H. (1967) "The nature of mental states", reprinted in D. Chalmers (ed.) The Philosophy of Mind, Oxford: Oxford University Press (2002), pp.73-79.

Kim, J. (2005) Physicalism, or Something Near Enough, Princeton: Princeton University Press.

Kim, J. (1998) Mind in a Physical World: An Essay on the Mind-Body Problem and Mental Causation, Cambridge: MIT Press.

Kim, J. (1991) Supervenience and Mind, Cambridge: Cambridge University Press.

Lewis, D. (1983) "New work for a theory of universals", Australasian Journal of Philosophy, Vol.61, pp.343-377. 
Libet, B. (1996) "Neural time factors in conscious and unconscious mental functions", in S. Hamerhoff, et al., (eds.) Toward a Science of Consciousness, Cambridge: MIT Press, pp.156-171.

McLaughlin, B. (1995) "Varieties of supervenience", in E. Savellos, and U. Yalcin, (eds.) Supervenience, Cambridge: Cambridge University Press, pp.16-59.

Mele, A. (1997) "Agency and mental action", Philosophical Perspectives, Vol.11, pp.231-249.

Menzies, P. (2003) "The causal efficacy of mental states", in S. Walter and H. Heckman, (eds.) Physicalism and Mental Causation, Exeter: Imprint Academic, pp.195-224.

Schlosser, M. (2007) "Basic deviance reconsidered”, Analysis, Vol.67, No.3, pp.186194.

Shoemaker, S. (1980) "Causality and properties", in his Identity, Cause and Mind, Oxford: Oxford University Press, 2003, pp.407-426. 
